\title{
LIMITS OF HYPERCYCLIC AND SUPERCYCLIC OPERATOR MATRICES
}

\author{
XIAOHONG CAO
}

\author{
(Received 20 June 2006; accepted 22 August 2006)
}

Communicated by J. J. Koliha

\begin{abstract}
An operator $A$ on a complex, separable, infinite-dimensional Hilbert space $H$ is hypercyclic if there is a vector $x \in H$ such that the orbit $\left\{x, A x, A^{2} x, \ldots\right\}$ is dense in $H$. Using the character of the analytic core and quasinilpotent part of an operator $A$, we explore the hypercyclicity for upper triangular operator matrix

$$
M_{C}=\left(\begin{array}{cc}
A & C \\
0 & B
\end{array}\right)
$$

2000 Mathematics subject classification: 47A53, 47A55, 47A15.

Keywords and phrases: hyperbolicity, analytic core, quasi-nilpotent part, spectrum.
\end{abstract}

\section{Introduction}

Throughout this paper, let $H$ and $K$ be infinite-dimensional separable Hilbert spaces, let $B(H, K)$ denote the set of bounded linear operators from $H$ to $K$, and abbreviate $B(H, H)$ to $B(H)$. For an operator $A \in B(H)$, write $A^{*}, \sigma(A), \rho(A), \sigma_{a}(A)$, iso $\sigma(A)$ for the adjoint, spectrum, resolvent set, approximate point spectrum, and isolated points of the spectrum $\sigma(A)$, respectively. By $n(A)$ and $d(A)$ we denote the dimension of the kernel $N(A)$ and the codimension of the range $R(A)$. If both $n(A)$ and $d(A)$ are finite, then $A$ is called a Fredholm operator and the index of $A$ is defined by $\operatorname{ind}(A)=n(A)-d(A) . \quad A \in B(H)$ is said to be a Weyl operator if it is Fredholm of index 0 . Recall that the ascent $\operatorname{asc}(A)$ of an operator $A$ is the smallest nonnegative integer $p$ such that $N\left(A^{p}\right)=N\left(A^{p+1}\right)$. If such an integer does not exist we put $\operatorname{asc}(A)=\infty$. Analogously, the descent $\operatorname{des}(A)$ of $A$ is the smallest nonnegative $q$ such that $R\left(A^{q}\right)=R\left(A^{q+1}\right)$ and if such an integer does not exist we put $\operatorname{des}(A)=\infty$. It is well known that if $\operatorname{asc}(A)$ and $\operatorname{des}(A)$ are finite then

This work is supported by the Support Plan of The New Century Talented Person of the Education Ministry, P.R. China.

(C) 2009 Australian Mathematical Society 1446-7887/09 \$A2.00+0.00 
$\operatorname{asc}(A)=\operatorname{des}(A)$. If $A$ is Fredholm with $\operatorname{asc}(A)=\operatorname{des}(A)<\infty$, we call $A$ a Browder operator. Note that if $A$ is Browder then $A$ is Weyl. The Weyl spectrum $\sigma_{w}(A)$ and the Browder spectrum $\sigma_{b}(A)$ of $A$ are defined by $\sigma_{w}(A)=\{\lambda \in \mathbb{C}: A-\lambda I$ is not Weyl $\}$ and $\sigma_{b}(A)=\{\lambda \in \mathbb{C}: A-\lambda I$ is not Browder $\}$.

For $x \in H$, the orbit of $x$ under $A$ is the set of images of $x$ under successive iterates of $A$ :

$$
\operatorname{Orb}(A, x)=\left\{x, A x, A^{2} x, \ldots\right\} .
$$

A vector $x \in H$ is supercyclic if the set of scalar multiples of $\operatorname{Orb}(A, x)$ is dense in $H$, and $x$ is hypercyclic if $\operatorname{Orb}(A, x)$ is dense. A hypercyclic operator is one that has a hypercyclic vector. We define the notion of supercyclic operator similarly. We denote by $H C(H)(S C(H))$ the set of all hypercyclic (supercyclic) operators in $B(H)$ and by $\overline{H C(H)}(\overline{S C(H)})$ the norm-closure of the class $H C(H)(S C(H))$. Supercyclic operators were introduced by Hilden and Wallen in 1974 [13]. Many fundamental results regarding the theory of hypercyclic and supercyclic operators were established by Kitai in her thesis [14].

Hypercyclicity or supercyclicity has been studied by many authors ([2, 3, 12], and so on). In this paper, using the character of the analytic core and quasinilpotent part of an operator $A$, we explore the hypercyclicity or supercyclicity for operator $A$ and for upper triangular operator matrix

$$
M_{C}=\left(\begin{array}{cc}
A & C \\
0 & B
\end{array}\right) .
$$

\section{Main results}

For an operator $A \in B(H)$, the analytic core of $A$ is the subspace

$$
\begin{aligned}
K(A)= & \left\{x \in H: A x_{n+1}=x_{n}, A x_{1}=x,\left\|x_{n}\right\|\right. \\
& \left.\leq c^{n}\|x\|(n=1,2, \ldots) \text { for some } c>0, x_{n} \in H\right\},
\end{aligned}
$$

and the quasinilpotent part of $A$ is the subspace

$$
H_{0}(A)=\left\{x \in H: \lim _{n \rightarrow \infty}\left\|A^{n} x\right\|^{(1 / n)}=0\right\} .
$$

The spaces $K(A)$ and $H_{0}(A)$ are hyperinvariant under $A$ and satisfy $N\left(A^{n}\right) \subseteq$ $H_{0}(A), K(A) \subseteq R\left(A^{n}\right)$ for all $n \in \mathbb{N}$ and $A K(A)=K(A)$; see [1, 15, 16] for more information about these subspaces.

We say that $A$ has the single-valued extension property (SVEP) at $\lambda_{0}$ if, for every open neighborhood $U$ of $\lambda_{0}$, the only analytic function $f: U \rightarrow H$ which satisfies the equation $(A-\lambda I) f(\lambda)=0$ for all $\lambda \in U$ is the function $f \equiv 0$. We say that $A$ has the SVEP if $A$ has the SVEP at every $\lambda \in \mathbb{C}$.

Next, we shall consider the hypercyclicity or supercyclicity for the class of operators $A \in B(H)$ and the operator matrices

$$
M_{C}=\left(\begin{array}{cc}
A & C \\
0 & B
\end{array}\right)
$$


for which the condition $\operatorname{dim} K\left(A^{*}\right)<\infty$ holds. In what follows, we suppose that $A$ is not quasinilpotent and let $H(A)$ be the set of all complex-valued functions that are analytic in a neighborhood of the spectrum $\sigma(A)$ of $A$. For $f \in H(A)$, the operator $f(A)$ is defined by the well-known analytic calculus. We start with a lemma.

Lemma 2.1. Suppose that $K\left(A^{*}\right)=\{0\}$. If $f \in H(A)$ is not constant, then:

(1) $\sigma(A)=\sigma_{w}(A)$ is connected;

(2) $\operatorname{ind}(f(A)-\lambda I) \geq 0$ for each $\lambda \in \rho_{S F}(f(A))$, where $\rho_{S F}(f(A))=\{\lambda \in \mathbb{C}$, $f(A)-\lambda I$ is semi-Fredholm $\}$;

$\sigma_{w}(f(A))=f\left(\sigma_{w}(A)\right)=\sigma(f(A))$ is connected.

ProOF. (1) We only need to prove that $\sigma(A) \subseteq \sigma_{w}(A)$. Let $\lambda_{0} \in\left[\sigma(A) \backslash \sigma_{w}(A)\right]$. There are two cases to consider.

Case 1. Let $\lambda_{0} \neq 0$. Since $A^{*}-\lambda_{0} I$ is Weyl and $\{0\} \neq N\left(A^{*}-\lambda_{0} I\right) \subseteq K\left(A^{*}\right)$, it follows that $K\left(A^{*}\right) \neq\{0\}$, which is a contradiction.

Case 2. Let $\lambda_{0}=0$. Since $A-\lambda_{0} I=A$ is Weyl, using the semi-Fredholm perturbation theory, $A^{*}-\lambda I$ is Weyl if $0<|\lambda|$ is sufficiently small. But since

$$
N\left(A^{*}-\lambda I\right) \subseteq K\left(A^{*}\right)=\{0\},
$$

it follows that $A^{*}-\lambda I$ is invertible. Then $0 \in$ iso $\sigma\left(A^{*}\right)$. By [15, Theorem], $H=H_{0}\left(A^{*}\right) \oplus K\left(A^{*}\right)=H_{0}\left(A^{*}\right)$, which means that $A^{*}$ is quasinilpotent. Thus $A$ is quasinilpotent, contradicting the assumption that $A$ is not quasinilpotent.

From the foregoing, we know that $\sigma(A)=\sigma_{w}(A)$. Suppose that $\sigma(A)$ is not connected. Then $\sigma\left(A^{*}\right)$ is not connected. Let $\sigma\left(A^{*}\right)=\sigma \cup \tau$, where $\sigma, \tau$ are closed, $\sigma, \tau \neq \emptyset$ and $\sigma \cap \tau=\emptyset$. Define $f \in H\left(A^{*}\right)$ such that $f \equiv 1$ on $\sigma$ and $f \equiv 0$ on $\tau$. Put $P=f\left(A^{*}\right)$. Then $P^{2}=P, R(P)$ and $N(P)$ are closed, $A^{*}$-invariant subspaces and $\sigma\left(\left.A^{*}\right|_{R(P)}\right)=\sigma$ and $\sigma\left(\left.A^{*}\right|_{N(P)}\right)=\tau$. Since $K\left(A^{*}\right)=\{0\}$, it follows that $A^{*} F \neq F$ for each closed $A^{*}$-invariant subspace $F \neq\{0\}$ [17, Proposition 2]. Then $0 \in \sigma \cap \tau$, which is a contradiction, since $\sigma \cap \tau=\emptyset$. Thus $\sigma(A)=\sigma_{w}(A)$ is connected.

(2) Since $N\left(A^{*}-\lambda I\right)=\{0\}$ for all $\lambda \neq 0, A$ has the SVEP. By [6, Theorem 1.5], $f\left(A^{*}\right)=f(A)^{*}$ has the SVEP. Therefore, $\operatorname{ind}(f(A)-\lambda I) \geq 0$ for each $\lambda \in$ $\rho_{S F}(f(A))$ by [9, Corollary 12].

(3) Applying (2) and [18, Theorem 3.6], we know that

$$
\sigma_{w}(f(A))=f\left(\sigma_{w}(A)\right)=f(\sigma(A))=\sigma(f(A))
$$

is connected.

If $K\left(A^{*}\right)=\{0\}$, then for any $f \in H(A)$,

$$
\sigma(f(A))=f(\sigma(A))=f\left(\sigma_{w}(A)\right)=\sigma_{w}(f(A))=\sigma_{b}(f(A))
$$

is connected. In this case, if $|f(\lambda)|=1$ for some $\lambda \in \sigma(A)$, then $f(\lambda) \in \sigma_{w}(f(A)) \cap$ $\partial \mathrm{D}$. Since $\sigma_{w}(f(A))$ and $\partial \mathrm{D}$ are connected, $\sigma_{w}(f(A)) \cup \partial \mathrm{D}$ is connected. If $\overline{H_{0}(A)}=H$, by $K\left(A^{*}\right) \subseteq H_{0}(A)^{\perp}[15]$, then $K\left(A^{*}\right)=\{0\}$. Using [12, Theorems 2.1 and 3.3], we have the following result. 
TheOrEm 2.2. Suppose that $K\left(A^{*}\right)=\{0\}$ or $\overline{H_{0}(A)}=H$. Then:

(1) $A \in \overline{H C(H)}$ if and only if there exists $\lambda \in \sigma(A)$ such that $|\lambda|=1$;

(2) $A \in \overline{S C(H)}$;

(3) for any $f \in H(A), f(A) \in \overline{H C(H)}$ if and only if there exists $\lambda \in \sigma(A)$ such that $|f(\lambda)|=1$;

(4) $f(A) \in \overline{S C(H)}$ for any $f \in H(A)$.

Corollary 2.3. Suppose that $K(A)=\{0\}$ and $K\left(A^{*}\right)=\{0\}$. Then:

(1) $A \in \overline{H C(H)}$ if and only if $A^{*} \in \overline{H C(H)}$, if and only if there exists $\lambda \in \sigma(A)$ such that $|\lambda|=1$;

(2) $A \in \overline{S C(H)}$ and $A^{*} \in \overline{S C(H)}$;

(3) for any $f \in H(A), f(A) \in \overline{H C(H)}$ if and only if $f\left(A^{*}\right) \in \overline{H C(H)}$, if and only if there exists $\lambda \in \sigma(A)$ such that $|f(\lambda)|=1$;

(4) $f(A) \in \overline{S C(H)}$ and $f\left(A^{*}\right) \in \overline{H C(H)}$ for any $f \in H(A)$.

The hypercyclicity (or supercyclicity) for operator matrices has been studied in [2]. In the following results, we continue this work.

Theorem 2.4. Suppose that $\operatorname{dim} K\left(A^{*}\right)<\infty$. Then the following statements are equivalent:
(1) $M_{0}=\left(\begin{array}{cc}A & 0 \\ 0 & B\end{array}\right) \in \overline{H C(H \oplus K)}$;
(2) $\quad M_{C}=\left(\begin{array}{ll}A & C \\ 0 & B\end{array}\right) \in \overline{H C(H \oplus K)}$ for each $C \in B(K, H)$;
(3) $\quad M_{C}=\left(\begin{array}{ll}A & C \\ 0 & B\end{array}\right) \in \overline{H C(H \oplus K)}$ for some $C \in B(K, H)$.

PROOF. We only prove the equivalence between (2) and (3), and so we only need to prove that (3) implies (2). Suppose that $M_{C_{0}} \in \overline{H C(H \oplus K)}$. Using [12, Theorem 2.1], we will prove that:

(a) $\sigma_{w}\left(M_{C}\right) \cup \partial \mathrm{D}$ is connected for each $C \in B(K, H)$.

We claim that $\sigma_{w}\left(M_{C}\right)=\sigma_{w}\left(M_{C_{0}}\right)$. If fact, let $M_{C}-\lambda_{0} I$ be Weyl. Then $A-\lambda_{0} I$ is upper semi-Fredholm, $B-\lambda_{0} I$ is lower semi-Fredholm and $d\left(A-\lambda_{0} I\right)<\infty$ if and only if $n\left(B-\lambda_{0} I\right)<\infty$. Using the perturbation theory of semi-Fredholm operators and the fact that $A^{*}-\lambda_{0} I$ is lower semi-Fredholm, there exists $\epsilon>0$ such that $A^{*}-\lambda I$ is lower semi-Fredholm, $\lambda \neq 0$ and $\operatorname{ind}\left(A^{*}-\lambda I\right)=\operatorname{ind}\left(A^{*}-\lambda_{0} I\right)$ if $0<\left|\lambda-\lambda_{0}\right|<\epsilon$. Since $N\left(A^{*}-\lambda I\right) \subseteq K\left(A^{*}\right)$, it follows that $n\left(A^{*}-\lambda I\right)<\infty$, which implies that $A^{*}-\lambda I$ is Fredholm. Then $A-\lambda_{0} I$ is Fredholm and hence $B-\lambda_{0} I$ is Fredholm. Therefore $M_{C_{0}}-\lambda_{0} I$ is Fredholm with ind $\left(M_{C_{0}}-\lambda_{0} I\right)=$ $\operatorname{ind}\left(M_{C}-\lambda_{0} I\right)=0$, that is, $M_{C_{0}}-\lambda_{0} I$ is Weyl. Then $\sigma_{w}\left(M_{C_{0}}\right) \subseteq \sigma_{w}\left(M_{C}\right)$. The case $\sigma_{w}\left(M_{C}\right) \subseteq \sigma_{w}\left(M_{C_{0}}\right)$ has the same proof. Then $\sigma_{w}\left(M_{C}\right) \cup \partial \mathrm{D}=\sigma_{w}\left(M_{C_{0}}\right) \cup \partial \mathrm{D}$ is connected for every $C \in B(K, H)$.

(b) $\sigma\left(M_{C}\right)=\sigma_{b}\left(M_{C}\right)$ for every $C \in B(K, H)$.

Let $M_{C}-\lambda_{0} I$ be Browder. Then both $A-\lambda_{0} I$ and $B-\lambda_{0} I$ are Fredholm and $\operatorname{asc}\left(A-\lambda_{0} I\right)<\infty, \operatorname{des}\left(B-\lambda_{0} I\right)<\infty$. Using the perturbation theory of 
semi-Fredholm operators again, there exists $\epsilon>0$ such that $A^{*}-\lambda I$ is Fredholm, $A^{*}-\lambda I$ is surjective, and $\operatorname{ind}\left(A^{*}-\lambda I\right)=\operatorname{ind}\left(A^{*}-\lambda_{0} I\right)$ if $0<\left|\lambda-\lambda_{0}\right|<\epsilon$. Since

$$
N\left(A^{*}-\lambda I\right) \subseteq K\left(A^{*}\right) \text { and } \operatorname{dim} K\left(A^{*}\right)<\infty,
$$

it follows that $A^{*}-\lambda I$ is bounded from below if $0<\left|\lambda-\lambda_{0}\right|$ is sufficiently small (less than $\epsilon$ ). Then $A^{*}-\lambda I$ is invertible if $0<\left|\lambda-\lambda_{0}\right|$ is sufficiently small. This implies that $\lambda_{0} \notin \operatorname{acc} \sigma(A)$. Then $A-\lambda_{0} I$ is Browder [10, Theorem 4.7]. Therefore $B-\lambda_{0} I$ is Browder and hence $M_{C_{0}}-\lambda_{0} I$ is Browder. Since $M_{C_{0}} \in \overline{H C(H \oplus K)}$, $\sigma\left(M_{C_{0}}\right)=\sigma_{b}\left(M_{C_{0}}\right)$. Then $A-\lambda_{0} I$ is injective and $B-\lambda_{0} I$ is surjective. But since both $A-\lambda_{0} I$ and $B-\lambda_{0} I$ are Browder, it follows that both $A-\lambda_{0} I$ and $B-\lambda_{0} I$ are invertible. Then $M_{C}-\lambda_{0} I$ is invertible, which proves that $\sigma\left(M_{C}\right)=\sigma_{b}\left(M_{C}\right)$ for every $C \in B(K, H)$.

(c) For every $C \in B(K, H)$, ind $\left(M_{C}-\lambda I\right) \geq 0$ for each $\lambda \in \rho_{S F}(A)$.

In fact, if $M_{C}-\lambda_{0} I$ is semi-Fredholm with $\operatorname{ind}\left(M_{C}-\lambda_{0} I\right) \leq 0$, then $A-\lambda_{0} I$ is Fredholm (see the proof of (a) above). By [4, Theorem 2.1], $B-\lambda_{0} I$ is upper semiFredholm. Thus $M_{C_{0}}-\lambda_{0} I$ is semi-Fredholm with

$$
\operatorname{ind}\left(M_{C_{0}}-\lambda_{0} I\right)=\operatorname{ind}\left(M_{C}-\lambda_{0} I\right)<0 .
$$

It is in contradiction to the fact that $M_{C_{0}} \in \overline{H C(H \oplus K)}$.

REMARK 2.1.

(1) Theorem 2.4 holds for the case of supercyclicity.

(2) The condition $\operatorname{dim} K\left(A^{*}\right)<\infty$ is essential in Theorem 2.4. For example, let $H=K=\ell_{2}$ and $A, B, C \in B\left(\ell_{2}\right)$ be defined by

$$
\begin{gathered}
A\left(x_{1}, x_{2}, x_{3}, \ldots\right)=\left(0, x_{1}, 0, x_{2}, 0, x_{3}, \ldots\right), \\
B\left(x_{1}, x_{2}, x_{3}, \ldots\right)=\left(x_{2}, x_{4}, x_{6}, \ldots\right), \\
C\left(x_{1}, x_{2}, x_{3}, \ldots\right)=\left(0,0, x_{1}, 0, x_{3}, 0, x_{5}, \ldots\right) .
\end{gathered}
$$

Then:

(i) $K\left(A^{*}\right)=K(B)=H$, then $\operatorname{dim} K\left(A^{*}\right)=\infty$;

(ii) $M_{0}=\left(\begin{array}{cc}A & 0 \\ 0 & B\end{array}\right) \in \overline{H C(H \oplus K)}$;

(iii) $M_{C} \notin \overline{H C(H \oplus K)}$.

In fact, we can prove that $M_{C}$ is bounded from below, but $M_{C}$ is not invertible. This means that there exists $\lambda \in \rho_{S F}\left(M_{C}\right)$ such that ind $\left(M_{C}-\lambda I\right)<0$. Then we

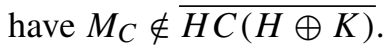

(3) Theorem 2.4 may fail if the assumption $\operatorname{dim} K(A)<\infty$ holds. For example, let $A \in B(H)$ be defined in (2) in this remark. We claim that $K(A)=\{0\}$. In fact, let $y=\left(y_{1}, y_{2}, y_{3}, \ldots\right) \in K(A)$. Using the definition of $K(A)$, there exists $\left\{x_{n}\right\} \subseteq H$ such that $A x_{n+1}=x_{n}$ and $A x_{1}=y$. Then $A^{n} x_{n}=y$ for any $n \in \mathbb{N}$. Let $x_{n}=\left(x_{n 1}, x_{n 2}, x_{n 3}, \ldots\right)$. For any $n \in \mathbb{N}$, the $n$th component of $A^{n} x_{n}$ is 0 . This proves that for $n \in \mathbb{N}, y_{n}=0$. Then $y=0$. Therefore $K(A)=\{0\}$. But the result in Theorem 2.4 fails. 
Example 2.1. Let $H=K=\ell_{2}$ and let $A \in B(H)$ and $B \in B(K)$ be defined by

$$
\begin{gathered}
A\left(x_{1}, x_{2}, x_{3}, \ldots\right)=\left(x_{2}, x_{4}, x_{6}, \ldots\right), \\
B\left(x_{1}, x_{2}, x_{3}, \ldots\right)=\left(0, x_{1}, 0, x_{2}, 0, x_{3}, \ldots\right),
\end{gathered}
$$

then $K\left(A^{*}\right)=\{0\}$ and $\left(\begin{array}{cc}A & 0 \\ 0 & B\end{array}\right) \in \overline{H C(H \oplus K)}$, therefore $M_{C} \in \overline{H C(H \oplus K)}$ for every $C \in B(K, H)$.

The equivalent definition of $K(A)$ is:

$$
K(A)=\left\{x \in H: \text { there exists }\left(x_{n}\right)_{n=1}^{\infty} \subseteq H \text { such that } A x_{1}=x, A x_{n+1}=x_{n},\right.
$$

(for any $n \in \mathbb{N}$ ), and $\left\{\left\|x_{n}\right\|^{(1 / n)}\right\}_{n=1}^{\infty}$ is bounded\}.

LEMMA 2.5. Suppose that $K(A)$ is closed. If for each eigenspace $N(A-\lambda I)$ of finite dimension, $K(A) \cap H_{0}(A-\lambda I)$ is closed, then asc $(A-\lambda I)<\infty$ for any $\lambda \in \mathbb{C}$ such that $A-\lambda I$ is upper semi-Fredholm.

Proof. Let $K(A) \neq\{0\}$ and suppose that $A_{1}=\left.A\right|_{K(A)}$. Then $A_{1}$ is surjective.

Let $\lambda_{0} \in \mathbb{C}$ such that $A-\lambda_{0} I$ is upper semi-Fredholm. Without loss of generality, let $\lambda_{0} \notin \sigma_{a}(A)$. If $\lambda_{0}=0$, since $K(A) \cap H_{0}(A)=H_{0}\left(A_{1}\right)$ is closed, we know that $A_{1}$ has the SVEP at $\lambda_{0}$. Then $n\left(A_{1}\right) \leq d\left(A_{1}\right)=0$ [9, Corollary 11], which means that $A_{1}$ is invertible. Then there exists $\epsilon>0$ such that $N(A-\lambda I)=N\left(A_{1}-\lambda I\right)=\{0\}$ if $0<|\lambda|<\epsilon$. Since $A$ is upper semi-Fredholm, $A-\lambda I$ is upper semi-Fredholm if $0<|\lambda|$ is sufficiently small. Then $A-\lambda I$ is bounded from below, that is, $0 \in\left[\right.$ iso $\left.\sigma_{a}(A) \cup \rho_{a}(A)\right]$. Therefore $\operatorname{asc}\left(A-\lambda_{0} I\right)<\infty$. In what follows, we suppose that $\lambda_{0} \neq 0$.

(a) For any $m \in \mathbb{N}, N\left[\left(A-\lambda_{0} I\right)^{m}\right] \subseteq K(A)$.

Let $x \in N\left[\left(A-\lambda_{0} I\right)^{m}\right]$, that is, $\left(A-\lambda_{0} I\right)^{m} x=0$. Then there exists a polynomial $P(\cdot)$ such that $\lambda_{0}^{m} x=A P(A) x, x=A\left[\left((P(A)) /\left(\lambda_{0}^{m}\right)\right) x\right]$. Let

$$
c=\left\|\left((P(A)) /\left(\lambda_{0}^{m}\right)\right)\right\|+1, \quad x_{1}=\left((P(A)) /\left(\lambda_{0}^{m}\right)\right) x, \quad x_{n}=\left[(P(A)) /\left(\lambda_{0}^{m}\right)\right]^{n} x,
$$

for all $n \in \mathbb{N}$. Then $A x_{1}=x, A x_{n+1}=x_{n}$, and $\left\|x_{n}\right\| \leq c^{n}\|x\|$, which implies that $x \in K(A)$. Therefore, $\alpha\left(A-\lambda_{0} I\right)=\alpha\left(A_{1}-\lambda_{0} I\right)$.

(b) $K(A) \cap R\left(A-\lambda_{0} I\right)=R\left(A_{1}-\lambda_{0} I\right)$.

For any $y \in K(A) \cap R\left(A-\lambda_{0} I\right)$, let $y=\left(A-\lambda_{0} I\right) x_{0}$. Since $y \in K(A)=A K(A)$, there exists $y_{0} \in K(A)$ such that $\left(A-\lambda_{0} I\right) x_{0}=A y_{0}$. Then

$$
x_{0}=A\left[\left(x_{0}+y_{0}\right) /\left(\lambda_{0}\right)\right] .
$$

Using the definition of $K(A)$, there exist $c>0$ and $\left\{y_{n}\right\}_{n=1}^{\infty} \subseteq X$ such that $A y_{1}=y_{0}, A y_{n+1}=y_{n}$ and $\left\|y_{n}\right\| \leq c^{n} \cdot\left\|y_{0}\right\|(\forall n \in \mathbb{N})$.

Let

$$
x_{1}=\left(\left(x_{0}+y_{0}\right) / \lambda_{0}\right), \quad x_{n}=\left(\left(x_{0}+y_{0}\right) / \lambda_{0}^{n}\right)+\left(y_{1} / \lambda_{0}^{n-1}\right)+\cdots+\left(y_{n-1} / \lambda_{0}\right) .
$$


Then $A x_{1}=x_{0}, A x_{2}=x_{1}, \ldots, A x_{n+1}=x_{n}$ and

$$
\begin{aligned}
\left\|x_{n}\right\| & =\left\|\frac{x_{0}+y_{0}}{\lambda_{0}^{n}}+\frac{y_{1}}{\lambda_{0}^{n-1}}+\cdots+\frac{y_{n-1}}{\lambda_{0}}\right\| \\
& \leq \frac{1}{\left|\lambda_{0}\right|^{n}}\left[\left\|x_{0}\right\|+\left\|y_{0}\right\|+\left|\lambda_{0}\right| \cdot\left\|y_{1}\right\|+\cdots+\left|\lambda_{0}\right|^{n-1} \cdot\left\|y_{n-1}\right\|\right] \\
& \leq \frac{1}{\left|\lambda_{0}\right|^{n}}\left[\left\|x_{0}\right\|+\left\|y_{0}\right\|+\left|\lambda_{0}\right| \cdot c \cdot\left\|y_{0}\right\|+\cdots+\left|\lambda_{0}\right|^{n-1} \cdot c^{n-1} \cdot\left\|y_{0}\right\|\right] \\
& \leq \frac{1}{\left|\lambda_{0}\right|^{n}}\left\|x_{0}\right\|+\frac{\left\|y_{0}\right\|}{\left|\lambda_{0}\right|^{n}}\left[1+\left|\lambda_{0}\right| c+\cdots+\left|\lambda_{0}\right|^{n-1} c^{n-1}\right] .
\end{aligned}
$$

If $\left|\lambda_{0}\right| \cdot c \leq 1$, then

$$
\begin{gathered}
\left\|x_{n}\right\| \leq \frac{1}{\left|\lambda_{0}\right|^{n}} \cdot\left\|x_{0}\right\|+\frac{\left\|y_{0}\right\|}{\left|\lambda_{0}\right|^{n}} \cdot n, \\
\left\|x_{n}\right\|^{(1 / n)} \leq \frac{1}{\left|\lambda_{0}\right|} \cdot\left\|x_{0}\right\|^{(1 / n)}+\frac{1}{\left|\lambda_{0}\right|} \cdot\left(n \cdot\left\|y_{0}\right\|\right)^{(1 / n)} .
\end{gathered}
$$

Since

$$
\lim _{n \rightarrow \infty}\left[\left(1 /\left(\left|\lambda_{0}\right|\right)\right) \cdot\left\|x_{0}\right\|^{(1 / n)}+\left(1 /\left(\left|\lambda_{0}\right|\right)\right) \cdot\left(n \cdot\left\|y_{0}\right\|\right)^{(1 / n)}\right]=2 /\left|\lambda_{0}\right|,
$$

it follows that $\left\{\left\|x_{n}\right\|^{(1 / n)}\right\}_{n=1}^{\infty}$ is bounded.

If $\left|\lambda_{0}\right| \cdot c>1$,

$$
\begin{aligned}
\left\|x_{n}\right\| & \leq \frac{1}{\left|\lambda_{0}\right|^{n}} \cdot\left\|x_{0}\right\|+\frac{\left\|y_{0}\right\|}{\left|\lambda_{0}\right|^{n}} \cdot \frac{1-\left|\lambda_{0}\right|^{n} \cdot c^{n}}{1-\left|\lambda_{0}\right| \cdot c} \\
& \leq \frac{1}{\left|\lambda_{0}\right|^{n}} \cdot\left\|x_{0}\right\|+\frac{\left\|y_{0}\right\|}{\left|\lambda_{0}\right|^{n}} \cdot \frac{\left|\lambda_{0}\right|^{n} \cdot c^{n}}{\left|\lambda_{0}\right| \cdot c-1} \\
& =\frac{1}{\left|\lambda_{0}\right|^{n}} \cdot\left\|x_{0}\right\|+\frac{\left\|y_{0}\right\|}{\left|\lambda_{0}\right| \cdot c-1} \cdot c^{n},
\end{aligned}
$$

then

$$
\left\|x_{n}\right\|^{(1 / n)} \leq \frac{1}{\left|\lambda_{0}\right|} \cdot\left\|x_{0}\right\|^{(1 / n)}+\left(\frac{\left\|y_{0}\right\|}{\left|\lambda_{0}\right| \cdot c-1}\right)^{(1 / n)} \cdot c .
$$

Also $\left\{\left\|x_{n}\right\|^{(1 / n)}\right\}_{n=1}^{\infty}$ is bounded. Using the equivalent definition of $K(A)$, we know $x_{0} \in K(A)$. Then $K(A) \cap R\left(A-\lambda_{0} I\right)=R\left(A_{1}-\lambda_{0} I\right)$. Hence $A_{1}-\lambda_{0} I$ is upper semi-Fredholm. Since $H_{0}\left(A_{1}-\lambda_{0} I\right)=K(A) \cap H_{0}\left(A-\lambda_{0} I\right)$ is closed, it follows that $A_{1}$ has the SVEP at $\lambda_{0}$. Then $\alpha\left(A-\lambda_{0} I\right)=\alpha\left(A-\lambda_{0} I\right)<\infty$.

Suppose that $K(A)=\{0\}$. Let $A-\lambda_{0} I$ be upper semi-Frehdolm. Then there exists $\epsilon>0$ such that $A-\lambda I$ is upper semi-Fredholm, $\lambda \neq 0$, if $0<\left|\lambda-\lambda_{0}\right|$ is sufficiently small. Since $N(A-\lambda I) \subseteq K(A), N(A-\lambda I)=\{0\}$. Then $A-\lambda I$ is bounded from below, and therefore $\lambda_{0} \in$ iso $\sigma_{a}(A)$. This also implies that $A$ has the SVEP at $\lambda_{0}$. Then $\operatorname{asc}\left(A-\lambda_{0} I\right)<\infty$. 
Let $\sigma_{d}(A)$ denote the surjective spectrum of $A$. From the statements in Remark 2.1, we know the result in Theorem 2.4 is not true if we suppose that $K(A)$ is closed. However, the following theorem holds.

THEOREM 2.6. Let $K(A)$ be closed. Suppose that for each eigenspace $N(A-\lambda I)$ of finite dimension, $K(A) \cap H_{0}(A-\lambda I)$ is closed.

(1) If $\sigma_{a b}\left(M_{C}\right)=\sigma_{a b}(A) \cup \sigma_{a b}(B)$ for any $C \in B(K, H)$ and $M_{C_{0}} \in \overline{H C(H \oplus K)}$ for some $C_{0} \in B(K, H)$, then $M_{C} \in \overline{H C(H \oplus K)}$ for any $C \in B(K, H)$.

(2) If $\sigma(A)=\sigma_{a}(A)$ or $\sigma(B)=\sigma_{d}(B)$, then the converse of (1) is true.

Proof. (1) (i) $\sigma_{w}\left(M_{C}\right) \cup \partial \mathrm{D}$ is connected for each $C \in B(K, H)$.

We claim that $\sigma_{w}\left(M_{C}\right)=\sigma_{w}\left(M_{C_{0}}\right)$. If fact, let $M_{C}-\lambda_{0} I$ be Weyl. Then $A-\lambda_{0} I$ is upper semi-Fredholm, $B-\lambda_{0} I$ is lower semi-Fredholm and $d\left(A-\lambda_{0} I\right)<\infty$ if and only if $n\left(B-\lambda_{0} I\right)<\infty$. Then $\operatorname{asc}\left(A-\lambda_{0} I\right)<\infty$. If $d\left(A-\lambda_{0} I\right)=\infty$, then by [5, Theorem 2.1] there exists $C_{1} \in B(K, H)$ such that $\lambda_{0} \notin \sigma_{a b}\left(M_{C_{1}}\right)$. Therefore $\lambda_{0} \notin \sigma_{a b}(A) \cup \sigma_{a b}(B)$, which implies that $n\left(B-\lambda_{0} I\right)<\infty$, which is a contradiction. Then both $A-\lambda_{0} I$ and $B-\lambda_{0} I$ are Fredholm. Therefore $M_{C_{0}}-\lambda_{0} I$ is Fredholm with $\operatorname{ind}\left(M_{C_{0}}-\lambda_{0} I\right)=\operatorname{ind}\left(M_{C}-\lambda_{0} I\right)=0$, that is, $M_{C_{0}}-\lambda_{0} I$ is Weyl. Then $\sigma_{w}\left(M_{C_{0}}\right) \subseteq \sigma_{w}\left(M_{C}\right)$. The case $\sigma_{w}\left(M_{C}\right) \subseteq \sigma_{w}\left(M_{C_{0}}\right)$ has the same proof. Then $\sigma_{w}\left(M_{C}\right) \cup \partial \mathrm{D}=\sigma_{w}\left(M_{C_{0}}\right) \cup \partial \mathrm{D}$ is connected for every $C \in B(K, H)$.

(ii) $\sigma\left(M_{C}\right)=\sigma_{b}\left(M_{C}\right)$ for every $C \in B(K, H)$.

Let $M_{C}-\lambda_{0} I$ is Browder. Then both $A-\lambda_{0} I$ and $B-\lambda_{0} I$ are Fredholm and $\operatorname{asc}\left(A-\lambda_{0} I\right)<\infty, \operatorname{des}\left(B-\lambda_{0} I\right)<\infty$. Since $\lambda_{0} \notin \sigma_{a b}\left(M_{C}\right), \operatorname{asc}\left(B-\lambda_{0} I\right)<\infty$, which means that $B-\lambda_{0} I$ is Browder. Then $A-\lambda_{0} I$ is Browder, and hence $\lambda_{0} \notin$ $\sigma_{b}\left(M_{C_{0}}\right)$. But since $\sigma\left(M_{C_{0}}\right)=\sigma_{b}\left(M_{C_{0}}\right)$, it follows that both $A-\lambda_{0} I$ and $B-\lambda_{0} I$ are invertible. Then $M_{C}-\lambda_{0} I$ is invertible. Therefore $\sigma\left(M_{C}\right)=\sigma_{b}\left(M_{C}\right)$ for every $C \in B(K, H)$.

(iii) For every $C \in B(K, H)$, ind $\left(M_{C}-\lambda I\right) \geq$ for each $\lambda \in \rho_{S F}(A)$.

In fact, if $M_{C}-\lambda_{0} I$ is semi-Fredholm with ind $\left(M_{C}-\lambda_{0} I\right) \leq 0$, then $A-\lambda_{0} I$ is upper semi-Fredholm with finite ascent. If $d\left(A-\lambda_{0} I\right)<\infty$, then by [4, Theorem 2.1] $B-\lambda_{0} I$ is upper semi-Fredholm. Thus $M_{C_{0}}-\lambda_{0} I$ is semi-Fredholm with

$$
\operatorname{ind}\left(M_{C_{0}}-\lambda_{0} I\right)=\operatorname{ind}\left(M_{C}-\lambda_{0} I\right)<0 .
$$

This contradicts the fact that $M_{C_{0}} \in \overline{H C(H \oplus K)}$. But if $d\left(A-\lambda_{0} I\right)=\infty$, using [5, Theorem 2.2], there exists $C_{1} \in B(K, H)$ such that $\lambda_{0} \notin \sigma_{a b}\left(M_{C_{1}}\right)$. Then $B-\lambda_{0} I$ is upper semi-Fredholm. Therefore $M_{C_{0}}-\lambda_{0} I$ is semi-Fredholm and further $\operatorname{ind}\left(M_{C_{0}}-\lambda_{0} I\right)=\operatorname{ind}\left(M_{C}-\lambda_{0} I\right)<0$. This again is a contradiction.

(2) Suppose that $\sigma(A)=\sigma_{a}(A)$ or $\sigma_{d}(A)=\sigma(B)$. For every $C \in B(K, H)$, the inclusion $\sigma_{a b}\left(M_{C}\right) \subseteq \sigma_{a b}(A) \cup \sigma_{a b}(B)$ is clear. For the converse inclusion, let $\lambda_{0} \notin$ $\sigma_{a b}\left(M_{C}\right)$, then $\lambda_{0} \notin \sigma_{a b}(A)$. Therefore $A-\lambda I$ is bounded from below if $0<\left|\lambda-\lambda_{0}\right|$ is sufficiently small. But since $\sigma_{a}(A)=\sigma(A)$, it follows that $\lambda_{0} \notin \operatorname{acc} \sigma(A)$. Then $A-\lambda_{0} I$ is Browder [10, Theorem 4.7]. Using the perturbation theory of semiFredholm operators and [4, Theorem 2.1], $\lambda_{0} \notin \sigma_{a b}(B)$. Then $\lambda_{0} \notin \sigma_{a b}(A) \cup \sigma_{a b}(B)$. The proof is complete. 
Corollary 2.7. If $\operatorname{dim} K(A)<\infty$ or $\operatorname{dim} K(A-\lambda I)<\infty$ for some $\lambda \in \mathbb{C}$, then the result in Theorem 2.6 is true.

In Lemma 2.5 and Theorem 2.6, we can modify the condition ' $K(A)$ is closed' to ' $K(A-\lambda I)$ is closed for some $\lambda \in \mathbb{C}$ '. It is well known that $K(A-\lambda I)=H$ is closed for any $\lambda \in \rho(A)$, leading to the following corollary.

Corollary 2.8. Suppose that for each eigenspace $N(A-\lambda I)$ of finite dimension, $H_{0}(A-\lambda I)$ is closed, then the result in Theorem 2.6 is true.

One such class which has attracted the attention of a number of authors is the set $H(P)$ of all operators $A \in B(H)$ such that for every complex number $\lambda$ there exists an integer $d_{\lambda} \geq 1$ for which

$$
H_{0}(A-\lambda I)=N\left[(A-\lambda I)^{d_{\lambda}}\right]
$$

holds. The class $H(P)$ contains the classes of subscalar, algebraically totally paranormal and transaloid operators on a Banach space, *-totally paranormal, M-hyponormal, $p$-hyponormal $(0<p<1)$ and log-hyponormal operators on a Hilbert space (see $[7,8,11]$ ). From Corollary 2.8 , we have the following results.

Corollary 2.9. If $A \in H(P)$, then the result in Theorem 2.6 is true.

Lemma 2.10. Suppose that $A^{*} \in H(P)$. Then $\sigma(A)=\sigma_{a}(A)$ and $\sigma_{a b}\left(M_{C}\right)=$ $\sigma_{a b}(A) \cup \sigma_{a b}(B)$ for every $B \in B(K)$ and for every $C \in B(K, H)$.

Proof. Let $A-\lambda I$ be bounded form below. Then $A^{*}-\lambda I$ is surjective. But since $A^{*}$ has the SVEP, it follows that $A^{*}-\lambda I$ is invertible. Then $A-\lambda I$ is invertible. This proves that $\sigma(A)=\sigma_{a}(A)$.

For any $C \in B(K, H)$ and for any $B \in B(K)$, the inclusion

$$
\sigma_{a b}\left(M_{C}\right) \subseteq \sigma_{a b}(A) \cup \sigma_{a b}(B)
$$

is clear. For the converse inclusion, let $\lambda \notin \sigma_{a b}\left(M_{C}\right)$; then $\lambda \notin \sigma_{a b}(A)$. Since $A^{*}$ has the SVEP at $\lambda, A-\lambda I$ is Browder. Then $B-\lambda I$ is upper semi-Fredholm with $\operatorname{asc}(B-\lambda I)<\infty$. This proves that $\sigma_{a b}\left(M_{C}\right)=\sigma_{a b}(A) \cup \sigma_{a b}(B)$.

Lemma 2.5 and Theorem 2.6 lead to the following result.

Corollary 2.11. Suppose that $A^{*} \in H(P)$ and $B \in B(K)$, then the following statements are equivalent:

(1) $M_{0} \in \overline{H C(H \oplus K)}$;

(2) $M_{C} \in \overline{H C(H \oplus K)}$ for some $C \in B(K, H)$;

(3) $M_{C} \in \overline{H C(H \oplus K)}$ for every $C \in B(K, H)$. 


\section{References}

[1] P. Aiena, Fredholm and Local Spectral Theory, with Applications to Multipliers (Kluwer Academic, Dordrecht, 2004).

[2] X. Cao, 'Weyl type theorem and hypercyclicity II', Proc. Amer. Math. Soc. 135 (2007), 1701-1708.

[3] _ 'Weyl type theorem and hypercyclic operarors', J. Math. Anal. Appl. 323 (2006), 267-274.

[4] X. Cao and B. Meng, 'Essential approximate point spectrum and Weyl's theorem for operator matrices', J. Math. Anal. Appl. 304 (2005), 759-771.

[5] X. Cao, 'Browder essential approximate point spectrum and hypercyclicity for operator matrices', Linear Algebra Appl. 426 (2007), 317-324.

[6] I. Colojoară and C. Foiaş, Theory of Generalized Spectral Operators (Gordon and Breach, New York, 1968).

[7] D. P. Duggal and S. V. Djordjević, 'Dunford's property and Weyl's theorem', Integral Equations Operator Theory 43 (2002), 290-297.

[8] D. P. Duggal, 'Polaroid operators satisfying Weyl's theorem', Linear Algebra Appl. 414 (2006), 271-277.

[9] J. K. Finch, 'The single valued extension property on a Banach space', Pacific J. Math. 58 (1975), $61-69$.

[10] S. Grabiner, 'Uniform ascent and descent of bounded operators', J. Math. Soc. Japan 34(2) (1982), 317-337.

[11] Y. M. Han and A. H. Kim, 'A note on *-paranormal operators', Integral Equations Operator Theory 49 (2004), 435-444.

[12] D. A. Herrero, 'Limits of hypercyclic and supercyclic operators', J. Funct. Anal. 99 (1991), 179-190.

[13] H. M. Hilden and L. J. Wallen, 'Some cyclic and non-cyclic vectors for certain operators', Indiana Univ. Math. J. 23 (1974), 557-565.

[14] C. Kitai, 'Invariant closed sets for linear operators', Dissertation, University of Toronto, 1982.

[15] M. Mbekhta, 'Généralisation de la décomposition de Kato aux opérateurs paranormaux et spectraux', Glasgow Math. J. 29 (1987), 159-175.

[16] M. Mbekhta and A. Ouahab, 'Opérateurs s-regulier dans un espace de Banach et théorie spectrale', Acta Sci. Math. (Szeged) 59 (1994), 525-543.

[17] C. Schmoeger, 'On isolated point of the spectrum of a bounded linear operator', Proc. Amer. Math. Soc. 117 (1993), 715-719.

[18] _ 'Ascent, descent and the Atkinson region in Banach algebras II', Ricerche Mat. XLII (1993), 249-264.

XIAOHONG CAO, College of Mathematics and Information Science, Shaanxi Normal University, Xi'an, 710062, People's Republic of China e-mail: xiaohongcao@snnu.edu.cn 